\title{
The effect of varied reinforcement training on behavior following incentive reduction
}

\author{
STEPHEN F. DAVIS ${ }^{2}$ AND ALVIN J. NORTH \\ SOUTHERN METHODIST UNIVERSITY
}

Three groups of rats were given 50 acquisition trials with large, varied, and small reward respectively. Following acquisition all Ss received 12 small reward trials. These 12 trials constituted an incentive reduction phase for the Ss trained under large and varied reward. The incentive reduction results showed a large decrement in performance for the Ss trained under large reward, while the Ss trained under varied reward showed only a very slight decrement in performance.

Crespi (1942) demonstrated that a shift from large to small reward resulted in performance that was, at least temporarily, inferior to the performance shown by a small reward control group. This phenomenon he called the "depression effect."

Subsequent researchers (Zeaman, 1949; Ehrenfreund \& Badia, 1962; DiLollo \& Lumsden, 1962; and DiLollo, 1964) confirmed Crespi's original finding. Other investigators (Bohmer \& Ison, 1966; Gonzalez, Gleitman, \& Bitterman, 1962) have shown that by giving partial reinforcement prior to the incentive reduction or that by gradually down-shifting the reward magnitude the depression effect may be lessened or possibly eliminated.

In accord with the Bohmer \& Ison results it would be predicted that giving varied reinforcement prior to an incentive reduction phase would also lessen or eliminate the decrement in performance typically observed. The present study was designed to investigate this prediction. Subjects

The Ss were 48 male albino rats, approximately 90 days old at the beginning of the experiment, purchased from the Holtzman Co., Madison, Wis. The Ss were maintained on 12-14 g Purina Lab Chow per day during the experiment. They were fed 15 min after the completion of the daily experimental session. Water was always available.

Apparatus

The apparatus consisted of a start box ( 4 in. $x 10 \mathrm{in.}$ $x 4$ in.), a runway ( 4 in. $x 32$ in. $x 4$ in.), and a goal box (4 in. $x 16$ in. $x 4$ in.), all made of wood. The start box was painted white and had six $3 / 4$ in. wide, $1 / 2$ in. high slats, mounted $3 / 4$ in. apart on the floor. The runway and goal box were painted black. Hinged hardware cloth lids covered the entire length of the apparatus. A goal cup was mounted 1 in. above the floor on the back wall of the goal box. Guillotine doors separated the start box from the runway and the runway from the goal box. Raising the starting door activated a timer which stopped when $S$ broke a photoelectric beam located 8 in. beyond the start door. Two other timers measured running times for the next 24 in. and 6 in. respectively. A fourth timer (goal time) measured running time in the $8 \mathrm{in}$. section in front of the goal cup. A fifth timer gave a total latency from the raising of the start door to the breaking of the last beam in the goal box.

\section{Procedure}

Upon receipt of the shipper all Ss were placed into individual cages and allowed ad lib food for four days. Then followed a week of pretraining. At the beginning of pretraining, deprivation was begun for all Ss. During pretraining Ss were handled and tamed, habituated to the reward pellets, and habituated to the unbaited apparatus (in groups of three and then singly). Ss were randomly divided into three groups: LR (large reward-15 45-mg Noyes pellets), VR (varied reward), and C (small reward control group-1 45-mg Noyes pellet). All Ss received 50 acquisition trials; the first 45 were given at the rate of three per day and the last five were given at the rate of one per day. The last five acquisition trials being given one per day in order to adjust $S s$ to the procedures of the incentive reduction phase. Group $L R$ received large reward on all 50 acquisition trials. Group VR also received 50 acquisition trials, half with large reward and half with small reward. The order for administering large and small reward acquisition trials to group VR was randomly determined for each $S$ with two restrictions. There could be no more than two of each type of trial in succession, and during the three-trial-per-day phase of acquisition there had to be an equal number of each type of trial on any two consecutive days. Group $\mathrm{C}$ received 50 small reward trials during acquisition. All three groups received a series of 12 trials (one per day) with small reward following acquisition. This constituted the incentive reduction phase for Groups $L R$ and VR. Resulis

Figure 1 shows the total time measure (in $\mathrm{ft}$ per sec) for terminal acquisition and incentive reduction.

Only results for total times are presented here because group differences on the part measures, while generally in agreement with those for total time, tended to be unreliable. Analysis of variance showed a significant groups effect $(F=9.04, d f=2 / 45, p<.01$ ), a significant trial blocks effect $(F=14.63, d f=6 / 270, p<.01)$, and a significant groups by trial blocks interaction $(F=3.18$, df $=12 / 270, p<.01$ ).

The significant interaction was further investigated by $t$ tests with the following significant differences being obtained. At terminal acquisition Group VR differed significantly grom Group $\mathrm{C}(\mathrm{t}=2.259,2.289,2.889,2.346$, $2.22 ; \mathrm{df}=31 ; \mathrm{p}<.05, .05, .01, .05, .05$, respectively). The only other significant difference was found at trial block 3 where Group VR differed significantly from Group C 


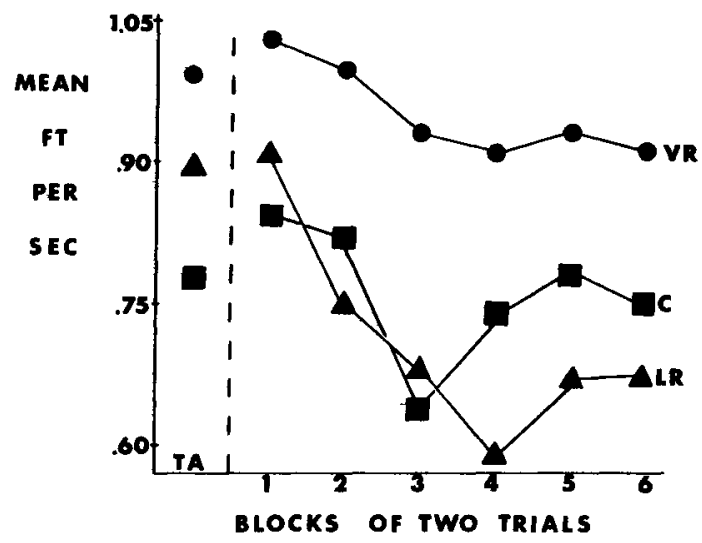

Fig. 1. Mean total speeds at terminal acquisition and during the incentive reduction phase.

$(t=2.639, d f=31, p<.01)$. No other significant differences were found.

\section{Discussion}

As can be seen from Fig. 1, the prediction that varied reward training would lessen the decrement in performance observed during incentive reduction was substantiated. Frustration theory (Amsel, 1958; Spence, 1960) would appear well suited to account for these results. First, it will be noted that at terminal acquisition Group VR was running faster than Groups LR and C. This terminal acquisition ordering of groups was consistent for all measures taken. For Group VR during acquisition it would be expected that after some number of trials the small reward trials would come to elicit a frustration reaction. Consequentially, Group VR would have, due to the frustration, an additional motivational factor present as compared with Groups LR and C. Hence, the terminal acquisition ordering.

Moreover, theoretically in Group VR the instrumental response became classically conditioned to the frustration-produced cues during acquisition. Therefore, during the incentive reduction phase any frustration responces elicited would serve to maintain the instrumental response longer and at a higher level for VR Ss. However, any frustration reactions emitted by LR Ss during incentive reduction would tend to disrupt their performance since the instrumental response had not been conditioned to such cues.

Even though the above interpretation appears fairly straightforward, one problem still remains unanswered. Concerning Group $\mathrm{C}$, there is no apparent reason for the disruption in performance shown during the incentive reduction phase (trial block 3). Theoretically, the experimental conditions for this group remained unchanged throughout the experiment. A methodological problem recently put forth by McHose and Ludvigson (1966) and Ludvigson and Sytsma (1967) may possibly offer an an- swer. They point out the possibility that rat Ss may, on reward and nonreward trials, exude either qualitatively or quantitatively different odors. Furthermore, Ludvigson and Sytsma suggest that rat Ss emit an unconditioned response to such odors left on prior nonreward trials which interferes with the approach response. It seems reasonable to assume that incentive reduction has odor effects similar to those of incentive omission (nonreward). Presumably in the present study Group LR Ss would be the most likely to lay down odors occasioned by incentive reduction. Due to randomization of the order for running Ss suring the experiment it is possible that on some days (e.g., trial block 3) several of the LR Ss were run consecutively and just prior to several Group C Ss. This would possibly account for the disruption in performance shown by Group C. Unfortunately the daily order for running Ss was not retained; therefore, no definite conclusion can be drawn.

One small difficulty with the odor explanation of the post shift data for Group $\mathrm{C}$ is that presumably all Ss during acquisition would have developed some habits of approaching in the face of such odors, because these presumably were laid down by Group VR Ss on small reward trials. On the other hand, these occasions were relatively infrequent and possibly not as potent as those odors laid down by LR Ss during incentive reduction.

\section{References}

AMSEL, A. The role of frustrative non-reward in non-continuous reward situations. Psychol Bull, 1958, 55, 103-119.

BOHMER, H. M., \& ISON, J. R. The effects of reward magnitude changes following partial reinforcement. (Paper Presented at Midwest. Psychol. Assoc., 1966).

CRESPI, L. P. Quantitative variation of incentive and performance in the white rat. Amer. J. Psychol, 1942, 55, 467-517.

DiLOLLO, V. Runway performance in relation to runway-goal-box similarity and changes in incentive amount. J. comp. physiol., Psychol., 1964, 58, 327-329.

DiLOLLO, V., \& LUMSDEN, J. The Crespi effect: a replication. Psychol Rep., 1962, 11, 25-26.

EHRENFREUND, D., \& BADIA, P. Response strength as a function of drive level and pre- and postshift incentive magnitude. J. exp. Psychol, 1962, 63, 468-471.

GONZALEZ, R. C., GleitMAN, H., \& BiTTERMAN, M. E. Some observations on the depression effect. J. comp. physiol. Psychol, $1962,55,578-581$.

LUDVIGSON, H. W., \& SYTSMA, D. The sweet smell of success: apparent double alternation in the rat. (Paper Presented at Midwest. Psychol. Assoc., 1967).

McHOSE, J. H., \& LUDVIGSON, H. W. Differential conditioning with nondifferential reinforcement. Psychon. Sci, 1966, 6, 485-486.

SPENCE, K. W. Behavior Theory and Learning. Prentice-Hall, Inc., Englewood Cliffs, 1960, Pp. 91-112.

ZEAMAN, D. Response latency as a function of the amount of reinforcement. J. exp. Psychol, 1949, 39, 466-483.

Notes

1. Supported by National Science Foundation Grant GB 2873 to the second author.

2. Present address: Department of Psychology, Texas Christian University. 